\title{
Racine et le mystère de la bonne rhétorique : repérage de discours dans La Thébaïde, Britannicus et Mithridate
}

\section{Francis Goyet}

\section{(2) OpenEdition Journals}

Édition électronique

URL : http://journals.openedition.org/rhetorique/98

DOI : $10.4000 /$ rhetorique.98

ISSN : 2270-6909

Éditeur

UGA Éditions/Université Grenoble Alpes

Édition imprimée

ISBN : 978-2-84310-255-4

\section{Référence électronique}

Francis Goyet, « Racine et le mystère de la bonne rhétorique : repérage de discours dans La Thébaïde, Britannicus et Mithridate », Exercices de rhétorique [En ligne], 1 | 2013, mis en ligne le 12 novembre 2013, consulté le 12 septembre 2020. URL : http://journals.openedition.org/rhetorique/98 ; DOI : https:// doi.org/10.4000/rhetorique.98

Ce document a été généré automatiquement le 12 septembre 2020.

\section{c) () (2) (2)}

Les contenus de la revue Exercices de rhétorique sont mis à disposition selon les termes de la Licence Creative Commons Attribution - Pas d'Utilisation Commerciale - Partage dans les Mêmes Conditions 4.0 International. 


\title{
Racine et le mystère de la bonne rhétorique : repérage de discours dans La Thébaïde, Britannicus et Mithridate
}

\author{
Francis Goyet
}

1 La rhétorique des personnages de Racine est sous le signe de l'échec, comme la critique l'a souligné de longue date. Ils ne cessent de vouloir persuader ceux à qui ils s'adressent, et ils y parviennent rarement. Un tel échec, en soi, n'est pas une surprise. D'une part, la rhétorique comme art ou technique n'a jamais garanti la victoire. D'autre part, si les personnages réussissaient plus souvent, il n'y aurait tout simplement pas de tragédie: Néron se réconcilierait avec Britannicus, et Étéocle avec Polynice. L'échec rhétorique est une donnée prévisible de l'univers tragique, c'est une contrainte imposée par le genre. Dès lors, il ne resterait plus qu'à illustrer par des études de cas la thématique d'un échec annoncé.

2 Mais il est évidemment tentant de penser que la fréquence même de ces échecs fait sens, un sens propre à Racine. Elle n'est pas aussi élevée par exemple chez Corneille, où une Pauline et une Émilie finissent par se convertir, par changer d'avis ou d'état d'esprit (cf. La Thébaïde ou T., 774, «Puisqu'il change votre âme »; Britannicus ou B., 1365 et 1766, «quel changement »). Si la récurrence de l'échec rhétorique est significative d'une vision proprement racinienne, il reste à dire ce qu'est cette vision, et là les avis divergent. Pour ma part, je proposerai ici de l'interpréter comme une méditation sur l'essence même de l'art de la parole, sur ses possibilités comme sur ses limites. En donnant à voir des échecs spectaculaires, Racine donnerait à comprendre à quoi ils tiennent, en s'adressant à des spectateurs avertis ou alertés. Visibilité de l'échec, mystère de la réussite: le spectaculaire initierait a contrario au mystère, le visible menant ainsi à l'invisible, de façon très classique. Dans cette hypothèse, ce ne sont pas les personnages qui sur scène se convertissent, ce sont les spectateurs qui changent de regard. Le spectacle de la mauvaise rhétorique nous apprend ce que doit être la bonne, 
tout comme, dans Britannicus, le spectacle de la tyrannie naissante renvoie à ce que devrait être une bonne «institution du Prince». Le mauvais roi parle du bon roi ; la mauvaise rhétorique, de la bonne rhétorique. Ce n'est pas là d'ailleurs une simple analogie, tant politique et parole sont intrinsèquement liées.

Le problème étant ainsi posé, l'étude de cas se limitera aux plus instructifs d'entre eux. Je regarderai d'abord un détail révélateur, la difficulté pour nombre de personnages d'anticiper l'objection qu'on pourrait leur faire. De cette micro-lecture je passerai ensuite à une macro-lecture, celle de La Thébaïde tout entière: le parti de la paix y anticipe, lui, la réconciliation des frères ennemis, mais celle-ci ne se réalise pas et ne donne donc pas lieu au discours classique nommé conciliatio.

\section{Anticiper les objections}

Dans un discours habituel, l'orateur fait presque toujours droit à l'objection ou aux objections de la partie adverse. En gros, deux cas de figure se présentent. Ou bien l'adversaire a déjà énoncé une objection, voire plusieurs. Ou bien l'orateur imagine ce que l'on pourrait lui objecter, sur le mode «On me dira que... » Dans les deux cas, l'objection et la réponse à celle-ci étant une réfutation (refutatio), elles prennent place de façon canonique après l'argumentation proprement dite (confirmatio). En revanche, elles se trouvent au tout début si le discours est d'emblée une réfutation des attaques de l'adversaire. C'est le cas de la première préface de Britannicus. Certains ont dit que je faisais Néron «trop cruel » (p. 254, § 1$)$ : suit la réponse. « D’autres ont dit, au contraire, que je l'avais fait trop bon» ( $\$ 2$, et $2^{\text {e }}$ objection), etc. Au début des $\S 5$ et 8 (p. 255), le style direct marqué par les guillemets restitue au jeu objection et réponse sa saveur originelle d'un dialogue entre personnages. "“Tout cela est inutile, disent mes censeurs. La pièce est finie au récit de la mort de Britannicus, et l'on ne devrait point écouter le reste." On l'écoute pourtant [...]. » La réponse de Racine est ici une réplique assez vive, qui évoque la stichomythie avec son effet d'écho et son côté cinglant, alors que les premières réfutations de cette préface étaient posées et raisonnées ${ }^{1}$.

5 Dans nos trois pièces, on trouve un certain nombre d'objections à l'intérieur du discours du personnage orateur. Soit comme seul exemple Pharnace à son frère Xipharès (Mithridate ou M. I, 5, 339-362). C'est un discours très politique et très réfléchi. Exorde: 339-342, qui se termine par l'attentio, «des soins plus importants». Argumentation: 345-354, Mithridate est à craindre, "redoutable", "Le péril est pressant », "Craignons », donc on agite le pathos de la crainte ou terreur, metus ou timor, en grec phobos (Aristote, Rhétorique, II, 5). L'argumentation a deux arguments : le péril est pressant a) «pour vous, pour moi » (345-350), b) il l'est aussi "pour la reine » (351-354). Conclusion et propositio : 359-362. La propositio est ici une proposition puisque l'on est dans le délibératif : allions-nous tous les deux contre le roi, c'est le seul moyen d'«assurer notre grâce »-assurer est le mot d'époque pour dire le contraire de la crainte.

6 Entre l'argumentation et la conclusion, on a l'objection et sa réponse, qui se situent donc ici à l'endroit canonique : 355-358. « Ne vous assurez point sur l'amour qu'il vous porte » : en supprimant la négative, on aurait l'objection au style direct, Xipharès dirait "Je m'assure [assurer, contraire de craindre] sur l'amour que notre père nous porte ", donc je ne crains rien. Réponse : «Sa jalouse fureur n'en sera que plus forte. » Nouvelle objection : «Vous avez la faveur des soldats » ou au style direct « Moi Xipharès, j’ai la 
faveur des soldats », réponse de Pharnace : «Et j'aurai des secours que je n'explique pas $^{2}$. » Les deux objections et leurs réponses font comme un petit dialogue à l'intérieur de la tirade de Pharnace, "vous me direz..., je vous répondrai..." ", dialogue lui aussi assez vif, aussi « pressant » que le péril. L'orateur est à la fin de son discours, il tente d'emporter par là les derniers remparts que pourrait lui opposer son auditeur. Il le presse dans ses retranchements, et ensuite veut croire que la place est prise ( $«$ M’en croirez-vous? "; même "vous me croirez» à B. 1373 comme marqueur de la conclusion). Conclusion: enfin, "courons ", « rendons-nous, vous et moi », ce qui est un bel exemple de "construction du nous»-cf. le même verbe courir à la fin de la célèbre scène du Cid, « Va, cours, vole et nous venge ». Avec ce verbe, l'orateur prend ses désirs pour la réalité : celui qu'il exhorte va s'empresser de faire ce qu'il propose, sans plus réfléchir. L'objection, en intégrant la voix et le point de vue de l'autre, a construit un nous, ce qui est le but standard de tout discours. Ce nous ou accord par hypothèse ne préexiste pas : pas de discours quand orateur et auditeur sont d'accord, d'emblée.

7 Loin de courir, l'autre ne bouge pas, qu'il soit Rodrigue ou Xipharès. Ce dernier va d'abord répondre à la réponse, ce qui s'appelait proprement au tribunal répliquer: «Je sais quel est mon crime, et je connais mon père » (363), quand vous avez l'air de croire que je ne sais pas à quel point il est redoutable. Ensuite, Xipharès va opposer une autre objection : "Quand mon père parait, je ne sais qu'obéir ». Je ne suis pas un naïf, et je pointe votre faiblesse à vous, votre manque de légitimisme. La différence entre l'objection de l'obéissance et celles qu'avait imaginées Pharnace est très importante. Sur le mode du "Vous me direz que ", l'orateur Pharnace avait avancé des objections faciles à lever, celles qu'on ne lui a pas faites et qu'il peut traiter avec une certaine condescendance - voir le mépris de formules comme "D'aucuns diront que... » Les objections réellement faites par l'adversaire sont plus coriaces, le réel étant comme on sait ce qui résiste. Face à l'objection réelle de l'obéissance, Pharnace fait machine arrière. Puisque sa proposition d'alliance est caduque, il la remplace par une autre, minimale, un pacte de non-agression : «en obéissant, ne nous trahissons pas » (374), c'est-à-dire, « $\mathrm{OK}$, obéissons à notre père, $\mathrm{j}$ 'intègre votre objection ».

On peut dès lors voir la scène de deux façons. Ou bien on aurait (i) un discours en forme, la tirade de Pharnace que j'ai analysée en commençant, puis (ii) une fin de nonrecevoir, et enfin (iii) une nouvelle proposition, moins ambitieuse, la non-agression (367). Ou bien on aurait un seul discours de Pharnace, qui couvrirait toute la scène, discours relancé par l'objection réelle de Xipharès. Les derniers propos de Pharnace sonnent en effet comme une péroraison de ce discours global (368-372), suivie de la nouvelle proposition (373-374). Quoi qu'il en soit, Pharnace est un réaliste, à l'école des Romains dont il est l'allié. Il a comme eux la prudentia, le sens de la stratégie, à preuve son anticipation des objections possibles. Mais il sait aussi intégrer l'objection réelle, s'adapter à la situation nouvelle, toujours en bon Romain et cette fois en bon tacticien. L'anticipation prépare l'adaptation. Il n'est qu'à moitié surpris par la réaction de Xipharès, ayant anticipé aussi l'échec de sa tirade. Sa proposition minimale est ainsi, en termes rhétoriques mais aussi militaires, une concession, mot qui veut dire au propre « céder du terrain face à l'ennemi », donc repli tactique.

9 Nous pouvons de là en venir à la situation la plus habituelle dans nos pièces, celle des objections non prévues qui jouent le rôle de relance, à l'instar de la réplique de Xipharès. À l'inverse de Pharnace, les personnages orateurs sont le plus souvent pris au dépourvu par la réaction de l'auditeur, qu'ils n'ont pas anticipée. Du coup, au lieu de 
s'adapter, ils s'entêtent; au lieu d'une concession, ils campent sur leurs positions. Si la différence est importante par rapport au cas de Pharnace, on peut pourtant procéder au même type d'analyse, et voir là encore la scène de deux façons. Au départ, la tirade d'un personnage n'imagine pas de réponse. Mais la réponse ou objection réelle jouant le rôle de relance, en répondant lui-même à la réponse, donc en répliquant, il se retrouve à construire un discours complet. $\mathrm{Ou}$ du moins pouvons-nous alors considérer comme un seul discours l'ensemble que constituent tirade, objection réelle et réponse à celleci. Le paradoxe à méditer est que c'est un discours non prévu. J'en donnerai deux exemples, au début de La Thébaïde.

Le premier est l'échange entre Antigone et Créon à propos du fils de celui-ci, Hémon ( $T$. I, 5). On peut en effet voir comme un seul discours les vers 249-271. Vu pour ainsi dire de haut, c'est une "scène de colère " (Aristote, Rhétorique, II, 2), comme il y a des « scènes de pitié ${ }^{3}$ ( $(R h .$, II, 8$)$. Vu de près, c'est une suite d'objections réelles :

ANTIGONE :

Créon, vous êtes père, et dans ces ennemis,

Peut-être songez-vous que vous avez un fils.

On sait de quelle ardeur Hémon sert Polynice.

11 Si c'était une tirade de Créon, celui-ci dirait : «Certes, je suis père, et je sais de quelle ardeur... » : ce serait alors une objection anticipée dans son discours. Voici maintenant la réponse à l'objection, sur le mode habituel du « Oui... Mais... » :

CRÉON :

Oui, je le sais, Madame, et je lui fais justice ;

Je le dois, en effet, distinguer du commun,

Mais c'est pour le haïr encor plus que pas un.

Et je souhaiterais, dans ma juste colère,

Que chacun le haït comme le hait son père.

12 La réponse se veut ou se croit définitive : circulez, il n'y a rien à discuter. Ma « juste colère » de père se comprend d'elle-même, elle est simplement posée, elle n'a pas besoin d'être appuyée ou justifiée par des arguments. C'est un pur cri. Mais de façon presque automatique, juste dans «juste colère » vaut signal, il en appelle au sens critique du spectateur et veut dire « hum, si cette colère est auto-proclamée juste, c'est qu'elle n'est pas si juste ", donc il faut l'argumenter, la justifier. Elle-même critique, Antigone réitère l'objection, qui est « Hémon est héroïque » :

ANTIGONE :

Après tout ce qu'a fait la valeur de son bras,

Tout le monde en ce point ne vous ressemble pas.

13 Nouvelle réponse à l'objection, le « bien » étant suivi de « mais » :

CRÉON :

Je le vois bien, Madame, et c'est ce qui m'afflige

Mais je sais bien à quoi sa révolte m'oblige ;

Et tous ces beaux exploits qui le font admirer,

C'est ce qui me le fait justement abhorrer [= abhorrer de façon juste, légitime].

14 Ce dernier vers retrouve le ton définitif de l'affirmation de la juste colère. Mais cette fois une telle affirmation est suivie de l'argumentation, réduite à un seul argument, d'ailleurs déjà donné par « sa révolte ». Hémon est certes héroïque, mais il est rebelle :

La honte suit toujours le parti des rebelles ;

Leurs grandes actions sont les plus criminelles,

Ils signalent leur crime en signalant leur bras,

Et la gloire n'est point où les rois ne sont pas. 

tranchant et définitif. Nouvelle objection, suivie d'une nouvelle réponse, cette fois sous la forme de la stichomythie :

ANTIGONE :

Écoutez un peu mieux la voix de la nature.

CRÉON :

Plus l'offenseur m'est cher, plus je ressens l'injure. l'offense " (Le Cid, I, 5, 285). Or dans la bouche de Don Diègue, il s'agissait justement de clore le bec de l'auditeur qu'est le jeune Rodrigue, à l'instar de la jeune Antigone - tous deux écoutant Amour et la nature :

Rodrigue. Le... Don Diègue. Ne réplique point, je connais ton amour,

Mais qui peut vivre infâme est indigne du jour,

Plus l'offenseur est cher, et plus grande est l'offense :

Enfin tu sais l'affront, et tu tiens la vengeance. [Suit la conclusion.] appelle un argument pathétique. Rhétorique, II, 2, § XV, 1379b13 : «On se fâche plutôt contre des amis que contre des indifférents; car on pense qu'il y a plutôt lieu d'en recevoir du bien que de n'en pas recevoir ${ }^{4}$. » Le pathos est l'argument suprême. Suit chez Créon l'équivalent d'une péroraison ou littéralement " après discours ", comme on dit avant-propos ("c'est trop parler»)-l'emportement dont parle ici Antigone désigne la colère quand elle dépasse les bornes (le De ira de Sénèque), quand elle n'est plus, précisément, une « juste colère » :

ANTIGONE :

Mais un père à ce point doit-il être emporté?

Vous avez trop de haine.

CRÉON :

Et vous trop de bonté.

C'est trop parler, Madame, en faveur d'un rebelle.

Au total, le cri initial de Créon est, de relance en relance, suivi de deux autres cris, de plus en plus haut et fort, sous forme de vers maximes, «Et la gloire n'est point où les rois ne sont pas », «Plus l'offenseur m'est cher, plus je ressens l'injure ».

La situation est très différente de la scène avec Pharnace. Certes, l'ensemble finit par ressembler à un discours, lequel justifie la colère face à la tentation du pardon. Mais on va d'affirmations définitives en affirmations définitives, où la voix de l'autre (l'objection) n'existe pas - la voix de l'autre étant moins celle d'Antigone que « la voix de la nature " ( $c f$. «la voix de mon vainqueur », 1224). Nulle polyphonie ou coexistence de " voix » au sens de Bakhtine, du moins dans la bouche du personnage. Ce qui existe pour lui, c'est son propre point de vue. De proche en proche, contraint et forcé, surpris et scandalisé, le personnage qui crie et clame en arrive à donner des arguments et des réponses, face au récalcitrant qu'il a en face de lui, cet auditeur tout aussi rebelle qu'Hémon. Vu de haut, cela ressemble à un discours habituel, polyphonique. Mais c'est pour mieux signifier que ce n'en est pas un. Pour pasticher Montaigne: "Nouvelle figure. Un orateur imprémédité et fortuit ${ }^{5}$.» Le problème est qu'un orateur qui ne prévoit pas n'est pas un orateur. Son but n'est pas de convaincre l'autre, mais de s'affirmer soi. Ni anticipation ni adaptation, ce n'est pas de la bonne rhétorique.

Mon autre exemple sera une scène plus développée, celle entre Polynice et Jocaste ( $T$. II, 3). Le type d'analyse que je viens de mener permet en effet de rendre compte du 
sentiment que bien des tirades sont simplement une narratio, un exposé de la situation. Rappelons que narratio désignait tout exposé des faits, aussi bien présents que passés, il ne faut pas limiter le terme aux seuls récits. Le schéma de base est donné par la fameuse scène du Cid. Don Diègue dit simplement à son fils : j'ai reçu un soufflet et n'ai pu répondre (narratio), donc venge-moi. Entre la narratio et sa conclusion, il n'est besoin d'aucune argumentation. Don Diègue prêche un converti, Rodrigue ne discute en rien, il est le public idéal de l'« orateur » convaincu de son bon droit, et qui n'a qu'à crier " au secours, on m'a souffleté !»Ce même schéma se retrouve avec Polynice. Enfin, sa mère Jocaste va cesser de l'interrompre, enfin il va pouvoir exposer la situation telle qu'il la voit et telle que la conclusion en découle évidemment (449-470). Enfin il peut crier contre son peuple, à la fin de sa narratio, "Quand il hait une fois, il veut haïr toujours » (470), ni plus ni moins que Créon a crié «Que chacun le haït comme le hait son père » (256). Cette narration est bien sûr orientée, elle a une visée argumentative, mais l'orateur ne voit pas le besoin d'aller plus loin, d'expliciter ses arguments, tant la conclusion lui parait couler de source. Le tout est une mise en demeure à Jocaste, avec ses questions rhétoriques qui appellent une réponse négative $(457,459)$. Mon peuple me hait, et vous ne seriez pas avec moi ? Mais où va-t-on, où va la monarchie si on prend le peuple pour juge ? Le propre du cri est de ne pas s'articuler en arguments.

Les réponses de Jocaste vont obliger Polynice à prendre en compte la résistance de son destinataire. Mais cela ne l'amènera pas comme Pharnace à des concessions. Les objections réelles et leurs réponses construisent une argumentation, avec une péroraison à 495-498. Vu de haut, on retrouve ainsi un ensemble qui est comme un discours en bonne et due forme. Le roi qui crie devient un roi qui se justifie. Cette sorte de discours global est le suivant. Le propos de Polynice est que, quant à lui, il tire sa légitimité du «sang » (480) elle-même sanctionnée par «le ciel» (451), et non comme Étéocle de l'amour du peuple pour son roi. Propos plus théorique que pratique : de « ce » peuple $(465,471$, quaestio finita, « hypothèse »), on passe via « un » peuple révolté, « une » troupe insolente $(457,459)$ à « le » peuple $(475,479,496,498$ et dernier ; $c f$. les «un» de 495-496). Polynice se place ou plutôt finit par se placer, dans son argumentation, sur le plan des principes, du côté de la quaestio infinita ou « thèse », qui est aussi la question de droit (an jure ?). La question de droit est : qui doit-on «prendre pour juge » (459), le «sang» ou le peuple (480) ? Réponse: le sang. Du coup, «ce» peuple devient rétrospectivement un cas particulier, une illustration du principe, sa révolte insolente ne prouve rien contre le principe, tout au contraire. Du fait ne se déduit pas le droit. Rétrospectivement, la tirade-narratio joue ainsi dans cette sorte de discours imprévu le rôle de toute narratio selon Quintilien, celui d'être une préparation de la suite c'est-à-dire de l'argumentation. Mais la différence est considérable. Le rétrospectif est ici une illusion, l'« orateur » n'avait pas prévu qu'il devrait expliciter ses arguments, qu'il devrait remonter au principe, donner comme dit Kant la maxime de son action. Il sort ses arguments comme on sort l'artillerie, face à la résistance imprévue d'un adversaire qu'il méprise. Cet orateur qui n'a pas prévu les objections n'imagine pas non plus de s'adapter à la nouvelle situation: pas de concessions en vue. - C'est pareil et différent avec Narcisse (B. IV, 4). Le flatteur s'adapte, il se sert des objections de Néron, qui sont la voix de Burrhus et de la vraie gloire, pour amener non pas lui-même mais Néron à changer d'opinion, à s'affirmer contre Burrhus. Jocaste avec ses objections à Polynice le braque, elle obtient qu'il s'enferre définitivement dans sa position légitimiste. Néron avec les mêmes objections s'enferre lui-même, tout aussi définitivement. L'habileté tactique de Narcisse est le pendant exact de l'absence 
d'habileté de Jocaste. En laissant parler l'autre, le flatteur cache l'art, Néron ne voit pas qu'on lui fait un discours global.

Péguy disait que tous les personnages de Racine sont blessants, volontairement ou non, dès qu'ils ouvrent la bouche. Polynice sinon Narcisse en est un exemple. Une réponse courtoise à sa mère aurait été de faire au moins semblant de faire droit à son objection, avec des politesses comme "Vous n'avez pas tort ", «J'en conviens volontiers », etc. Il est clair que ce n'est pas au programme. Comme le dit aussitôt Jocaste, Polynice ne veut rien « accorder » à sa mère (" N'accorderez-vous rien aux larmes d'une mère ? ", 503). Plutôt blesser que céder ou même concéder. Tout ce qu'il a bien voulu concéder, comme son frère d'ailleurs, c'est un délai ${ }^{6}$.

\section{La conciliatio ou discours de (ré)conciliation} donc la discorde a pour vous tant de charmes? "(499). Par rapport à Pharnace le Romain, si stratège, si réfléchi, on nous donne à voir des impulsifs, entièrement sujets à leur passion. Leur incapacité à anticiper toute objection permet au spectateur d'anticiper qu'ils ne sont pas près de se réconcilier, alors qu'un Pharnace raisonne aussitôt en termes de nouvelle alliance. Du coup, face à ces passionnés, les objections réelles sont contre-productives. Elles excitent au lieu de calmer. Jocaste et surtout Antigone sont, à l'évidence, fort peu diplomates. Elles croient plaider la paix, elles croient par leurs objections obliger à des concessions ou négociations. Elles ne font que braquer l'autre, "l'aigrir » (B. 357), le crisper sur ses positions par l'ivresse même de proclamer haut et fort ses arguments et ses principes. Antigone est surprise de voir chez Créon « à quel point son insolence monte » (291), mais c'est bien elle qui l'a poussé à monter le ton, qui a " aigr(i) (son) courroux » (T. 1431). Antigone du reste est comme lui un pur cri, elle est un aussi piètre orateur. Son but est tout autant de s'affirmer soi, et peu importe de blesser l'autre, d'où la stichomythie ou duel qui conclut leur échange. En termes d'époque, chacun de ces passionnés se voit sans doute comme un " magnanime ", une grande âme qui n'a pas la bassesse toute courtisane de faire des politesses et des concessions. Car s'affirmer comme Créon en pleine parrhèsia ou hardiesse de parole, c'est signer sa grandeur d'âme, une grandeur que les autres interprètent comme orgueil ou « superbe ${ }^{7} »$.

Si l'on voulait théoriser ces évidences, on pourrait tout au plus renvoyer la différence entre un Polynice et un Pharnace à l'opposition classique entre antinomie et hiérarchie. Polynice est dans l'antinomie absolue, le aut... aut. La légitimité dynastique est pour lui un principe indiscutable, qui exclut toute autre considération. À l'inverse, la hiérarchie est le monde conciliant du non solum... sed etiam, chaque « voix » y a sa place : quand on hiérarchise des priorités, on leur assigne un rang et une place, et choisir la première n'est pas mépriser les autres. La monarchie rêvée ou idéale est typiquement hiérarchique en ce sens, elle combine légitimité dynastique et amour du peuple pour son prince, alors que Polynice et Étéocle incarnent chacun de façon exclusive l'un ou l'autre aspect. L'amour du peuple était vu comme l'ornement de la légitimité dynastique, ou pour le dire en termes modernes la légitimité dynastique était 
nécessaire mais non suffisante, et l'amour du peuple, non nécessaire, était un " plus » qui faisait toute la gloire du monarque. Cette situation idéale est parfois bien réelle : un Henri IV selon Malherbe serait élu à une très grande majorité, si élection il devait y avoir8. Pour revenir au problème ponctuel de l'objection, « faire droit » à l'objection et donc à la voix de l'autre est ainsi une des formes que prend la hiérarchisation, qui est par définition intégrante quand l'antinomie est par définition excluante. C'est dire aussi que la bonne rhétorique que nous cherchons est, presque par définition là encore, du côté de la hiérarchisation.

Si la théorie de la réconciliation se conçoit aisément, c'est sa mise en œuvre pratique qui est le problème. Les traités de rhétorique du temps peuvent ici nous être de quelque secours, et pour ma part je suivrai Vossius (Rhetorice contracta, voir plus loin dans ce même numéro la section Atelier). Parmi les genres de discours qu'il décrit, se trouve le discours nommé conciliatio. De façon globale, la conciliatio fonctionne en couple d'opposés avec le discours de concitatio. Celui-ci consiste comme son nom l'indique à exciter à la sédition, donc à séparer plutôt qu'à unir. La recette est simple : l'orateur doit mettre en avant les torts subis (les « injures» ou injuriae), et susciter ainsi ira et odium, la colère c'est-à-dire le désir d'une juste vengeance, et la haine qui est quant à elle inextinguible, qui est la colère invétérée. La conciliatio est le discours inverse, pour faire oublier sinon pardonner les offenses. Il en est de deux sortes ${ }^{9}$. La première consiste à ramener à l'obéissance des troupes révoltées, dans une asymétrie entre l'orateur et la masse. La deuxième sorte vise à réconcilier deux partis ou parties de même taille et force. C'est la situation de La Thébaïde, où tout, à commencer par la gémellité, dit la symétrie et l'équilibre, ce que les Grecs nommaient l'isocratie ou l'isonomie, d'où sort l'impossibilité de trancher. Notons que la traduction de conciliatio par « réconciliation » cadre mieux avec le premier cas qu'avec le second. En rigueur de termes, on ne peut «ré» concilier Polynice et Étéocle, puisqu'ils se détestent depuis toujours.

Avant de poursuivre la lecture du traité, on peut déjà voir que toute La Thébaïde tend vers un tel discours de conciliatio. Le parti de la paix attend et nous fait attendre l'apothéose ou le miracle final d'une conciliation. Cela ressemblerait à ce que décrit Tite-Live $^{10}$. Les deux censeurs qui viennent d'être élus se détestent cordialement. Mais on profite de leur cérémonie d'installation pour les pousser à se serrer la main. L'idée de cérémonie religieuse est essentielle, la rhétorique combine rituel et sacré ${ }^{11}$. La scène se passe au Champ de Mars, les censeurs sont assis « sur leurs chaises curules auprès de l'autel de Mars ", autel sur lequel ils vont jurer, en présence des principaux du sénat et d'un grand nombre de citoyens romains. L'un des sénateurs prend d'abord la parole pour exhorter les deux élus à ne plus être ennemis. Son discours est interrompu par " mille voix " qui répètent la même prière. En réponse, les deux ennemis tiennent à tour de rôle un discours, où chacun se dit prêt à oublier sa haine, non sans avoir d'abord cité ses griefs contre l'autre, pour mieux montrer la grandeur du sacrifice qu'il consent sur l'autel de la patrie. "Alors, sur les instances de tous les assistants, ils se donnèrent la main, s'engagèrent à déposer et abjurer franchement leur haine, et furent conduits au Capitole au milieu d'applaudissements unanimes. » La cérémonie s'achève par ce geste rituel et encore aujourd'hui si médiatique, la poignée de mains entre anciens ennemis, telle celle de Rabin et Arafat réunis par le président américain Clinton. 
28 C'est une telle scène que Jocaste appelle de ses vœux, avec elle-même dans le rôle du maître de cérémonie. De même que Clinton poussa de façon assez visible Rabin et Arafat à se serrer la main "pour la photo ", de même Jocaste pousse Polynice dans les bras d'Étéocle, «Commencez, Polynice, embrassez votre frère, / Et montrez [...] » Les mots par lesquels Étéocle l'interrompt renvoient directement à cette idée de cérémonie quasi religieuse : «Hé, Madame ! à quoi bon ce mystère ?» (T. IV, 3, 999-1000). Ce mot de mystère est à la fois d'une ironie cruelle et d'une vérité profonde, à l'insu même d'Étéocle. Celui-ci veut dire évidemment qu'il s'agit là à ses yeux d'un faux mystère, que ce sont des mines et des détours puérils, des simagrées faciles à éventer. Il n'est pas dupe, on ne l'aura pas. Furetière, au mot mystère: « MYSTERE, se dit aussi des choses qui sont faciles. [...] Il y a des gens qui font mystère de tout, qui disent à l'oreille quelque chose, pour faire croire qu'il y a bien du mystère, qui font mystère de dire une chose connue de tout le monde. » Mais pour le spectateur, le mot qu'emploie Étéocle révèle, contre lui, tout le scandale de son attitude. Furetière: "MYSTERE, se dit aussi des sacrements, des cérémonies de l'Église. Il ne faut pas qu'un profane, qu'un excommunié participe à nos sacrés mystères ", et encore, au mot mystérieux : «Toutes les cérémonies, les ornements de l'Église renferment quelque chose de mystérieux. »

29 La remarque pour ainsi dire «méta » d'Étéocle fait capoter tout le processus, et dans le même temps nous le donne à voir - nous le révèle - comme processus. La cérémonie de la parole, quasi religieuse, que Jocaste mettait péniblement en place est tout à la fois une procédure et (comme à Éleusis) une procession, c'est-à-dire une progression, un chemin ou voie dans laquelle chacun peut entrer progressivement et librement, comme on entre dans la danse. Selon le concept de Dominique Maingueneau, c'est de façon typique une "co-construction ». Le cercle de la danse ou la cérémonie préexistent d'une certaine manière aux participants, et en même temps ont besoin de la bonne volonté de tous. Pas de danseurs sans danse, et inversement. Le rythme et le cercle appellent à participer, et la participation renforce le rythme et le cercle, dans une dynamique qu'alimente une série de bouclages et de récursivités. Selon une image encore plus familière, petit à petit la mayonnaise prend. La dynamique y va de pair avec la hiérarchisation. Dans l'histoire de Tite-Live, relève de la hiérarchisation le fait que chaque ennemi rappelle ses griefs contre l'autre, après que le sénateur ait marqué les griefs de Rome contre eux deux. Chacun est ainsi assigné à sa place, on n'a oublié personne, on a très soigneusement respecté les formes, tous traits du rituel, de la politesse ou de la rhétorique.

Étéocle quant à lui n'a pas le sens du mystère. Il ne se laisse pas impressionner par la cérémonie, avec lui rien ne prend. Interrompre comme il le fait le maitre de cérémonie est une impolitesse majeure, qui devait faire sursauter un spectateur de l'époque. C'est dire aussi que, en bonne rhétorique, le discours est lui-même une cérémonie, un processus qui amène le destinataire à se convertir, à changer d'avis voire d'état d'esprit, à accepter librement la place qu'on lui fait. Un tel processus est à la fois évident et sacré. C'est un processus évident : il n'a rien de mystérieux au sens de caché ou d'incompréhensible, cela ne relève pas du difficile. Mais c'est aussi un processus sacré : il faut y entrer progressivement, accepter de jouer le jeu, d'entrer dans la danse, d'y prendre sa place. Le collectif y prime sur l'individuel, comme la hiérarchisation sur l'antinomie. Le vocabulaire religieux s'impose donc pour en parler. La linéarité réglée d'un discours rhétorique en bonne et due forme est l'équivalent d'une liturgie. 
31 Le mot de mystère dans la bouche d'Étéocle est ainsi l'emblème de l'échec de la rhétorique, mais il dit aussi une vérité sur la réussite, du moins pour qui sait voir et entendre - pour qui est initié au mystère de la bonne rhétorique. La vérité sort ici de la bouche non des enfants, mais des méchants. Au moment même où Étéocle se moque de sa mère, le spectateur peut se moquer de lui, ou plutôt voir avec terreur et pitié comment parlent ceux qui font échouer la réconciliation si désirable, et par essence miraculeuse (« Madame, espérons tout de ce dernier miracle », T. 691). La réconciliation étant aux couleurs chrétiennes de l'oubli des offenses, le dispositif est celui de la Révélation. Parce que chrétiens, les spectateurs du temps «voient» les limites d'un païen, ils voient ce qui l'empêche d'être chrétien, et donc tout ce qui les sépare de lui. Britannicus n'est pas moins tendu par l'espoir d'une réconciliation. La terreur et la pitié y sont à leur comble avec le retour du mot mystère, cette fois dans la bouche de Burrhus, «Ce dessein s'est conduit avec plus de mystère » $(B . \mathrm{V}, 5,1619)$. Le banquet, collectif et convivial par définition, est le cadre idéal pour la même cérémonie tant attendue, tout comme les paroles de Néron levant sa coupe : mais ce nouveau maître du rituel est resté impassible quand Britannicus s'est effondré. Or le banquet dans l'Antiquité a une forte dimension religieuse ("dieux»,1625), et le monde chrétien en a toujours rapproché la Cène et donc la messe. Au mystère d'un banquet « eucharistique » succède ici, pour la moitié de la salle qui s'enfuit, l'échec du rituel, et pour la moitié qui reste le mystère atroce d'une collectivité de courtisans, calquant leur visage sur celui du nouveau maître. Là encore, ce n'est pas difficile, et c'est sacré, mais d'un sacré entièrement païen. Les courtisans communient, mais dans le mal. En fait de mystère, c'est ici celui de l'asservissement à la tyrannie, de la «servitude volontaire " (uoluntaria seruitus, Cicéron, $1^{\text {re }}$ Philippique ${ }^{12}$ ).

Pour revenir à $L a$ Thébaïde, la macro-lecture rhétorique de la pièce consiste à considérer le tout comme une montée vers la conciliatio, comme une préparation et anticipation de celle-ci. Le programme est tracé deux fois par Jocaste. Le premier est très ambitieux (I, 6, 300-302) :

Ciel, dispose à la paix le cœur de Polynice, Seconde mes soupirs, donne force à mes pleurs,

Et comme il faut enfin fais parler mes douleurs.

Primo, il faut « disposer » le cœur de l'auditeur à la réconciliation ou " paix ». Secundo, il faut que je pleure et soupire : ethos du supplex, automatique pour qui a une requête à présenter. Tertio ou "enfin ", Jocaste en viendra aux paroles, "parler ", elles-mêmes pathétiques, «douleurs » : étant émue par les malheurs de Thèbes, elle émouvra, selon la consigne de Quintilien. Ce beau programme est très classique, comme l'est l'invocation au Ciel. L'orateur va mettre en œuvre tous les moyens humains, c'est-àdire en l'espèce ceux de la rhétorique; et le Ciel, les moyens divins (la grâce). Car comme le répétaient tous les prédicateurs, seul Dieu peut véritablement toucher les cœurs, la gloire de la conversion en dernier ressort lui appartient.

Mais si nous prenons ce programme par la fin, l'échec est magistral. Le grand discours attendu de conciliatio n'a pas eu lieu, interrompu dans l'œuf par Étéocle. Auparavant, les larmes n'ont produit aucun effet: nous avons vu que Polynice n'a rien accordé «aux larmes d'une mère » (503). Ne reste plus que le premier élément du dispositif, disposer le cœur à la paix, donc travailler très en amont. Le programme ainsi réduit est réénoncé au vers 777 par Jocaste: "Bientôt ces cœurs de fer se verront adoucis». L'antithèse annonce que c'est désormais une mission quasi impossible, ou que si Jocaste 
y parvient, ce sera vraiment un miracle. Quoi qu'il en soit, c'est bien là un programme rhétorique. En effet, «adoucir» ou " apaiser » ou " désarmer », tous ces verbes de la pièce renvoient au pathos de l'apaisement ou lenitas, ce contre-feu au pathos de la colère (Aristote, $R$., II, 3). Aristote y décrit trois éléments : les motifs qui déclenchent le pathos de l'apaisement, et les deux personnes impliquées, celle qui s'apaise et celle envers qui on s'apaise. Le programme restreint de Jocaste porte sur le deuxième élément. Aristote le traite, comme toujours dans ses chapitres sur les pathè, en parlant de l'habitus ou disposition stable (grec hexis) de la personne à qui s'adresse l'orateur. Pour l'apaisement, les deux premiers habitus de sa liste sont les suivants. Tout d'abord sont apaisables ceux qui sont dans un état tranquille excluant la colère (1380b), parce qu'ils sont par exemple «dans un jour de bonheur, dans un moment de succès ", etc. Application probable, Junie à Néron: "Vos jours toujours sereins coulent dans les plaisirs » (B. 650-654), donc que votre majesté ne se mette pas en colère. Sont ensuite apaisables « ceux qui ont laissé passer du temps et ne s'emportent pas tout de suite, car le temps fait tomber la colère ", ou dans le latin de Vossius, "Qui inveteratam iram habent. Iram enim sedat tempus ${ }^{13}$ ", avec pour exemple chez lui la colère de Junon contre les Troyens dans l'Énéide, qui à la longue doit s'apaiser.

C'est sur ce deuxième habitus que table Jocaste. La « colère invétérée » est une façon de décrire la " haine » (« apaiser votre haine », T. 912). Par exemple, chez Tite-Live, Rome n'a pas seulement envers Capoue assiégée colère (le désir de châtier son passage dans le camp d'Hannibal) mais haine, qui ne sera saisie d'aucune pitié face aux malheurs des vaincus, alors que la colère, elle, s'apaiserait face aux malheurs (Aristote, Rh., II, 4, fin, 1382a). Un tel exemple peut laisser perplexe quant à la confiance dans l'efficacité du deuxième habitus d'Aristote, la haine comme colère invétérée redisant les " cœurs de fer ». Cet optimisme est pourtant bien celui du sénateur chez Tite-Live, face aux deux censeurs ennemis : «C'est une maxime bien connue, et qui est passée en proverbe à cause de sa vérité, que les amitiés doivent être immortelles, et les haines passagères ${ }^{14}$. " D'où l'intérêt du développement d'Étéocle sur la " haine obstinée » qui lie les deux jumeaux depuis leur cohabitation dans le ventre de leur mère (913-930). En bonne logique aristotélicienne, elles suscitent un dernier espoir de revirement miraculeux. Il devrait s'en déduire, justement, la fin de cette haine, leur rage devrait céder à la longueur de temps. C'est bien ce que craint Créon, qui compte comme antidote sur la vue de près pour raviver une haine qui à la longue menace de s'éteindre. En fait d'optimisme, prenons d'ailleurs garde que le texte même de Tite-Live parle plutôt d'obligation. Les haines doivent être passagères, comme Créon dit que la révolte de son fils l'oblige à la colère (260) ou, à Étéocle, «Vous devez, ce me semble, apaiser votre haine » $\left(912^{15}\right)$. Le pathétique a une bonne part de prescriptif. « Quittez, mon fils, quittez cette haine farouche» (780), "tout condamne vos haines» (1027) : c'est une prière de supplex mais aussi un ordre, comme chez Tite-Live, l'ordre très fort que donne la collectivité aux individus. De toute façon, dans l'Antiquité, l'attitude de suppliant contraint l'auditeur à faire un geste, à moins de se donner à voir comme inhumain (« inhumain » à 1021).

37 Tout ce travail en amont sur l'habitus des deux frères commence par échouer (III, 3, 685 et 688) : « Ce fils est insensible et ne m'écoute pas ; / [...] Il feint de s'apaiser, et devient plus sévère ». Mais ensuite, divine surprise, la mort du fils de Créon voit arriver un miracle : Créon devient, lui, « sensible » (771). De ce « si grand changement » (779), Jocaste aussitôt espère celui d'Étéocle, elle cherche à saisir l'occasion ou kairos par les cheveux. La temporalité rhétorique de la pièce est ainsi la suivante, dans une 
série espérée (et déçue) de réactions en chaîne. Le pathos à un temps $t$ produira un changement d'ethos au temps $t+1$ : touché par la mort de son fils, Créon changera d'ethos, d'attitude, l'ethos comme disposition passagère pouvant à son tour mener à cette disposition stable qu'est l'habitus. Puis, au temps $t+2$, Étéocle avec un peu de chance (et beaucoup de grâce divine) changera lui aussi d'ethos. Comme à Éleusis, une procession préalable au mystère se met ainsi en place. Bien qu'à l'extérieur du temple (c'est-à-dire avant la cérémonie de IV, 3), la procession est ponctuée par des "stations » elles-mêmes religieuses que sont ici les morts successives, clairement sacrificielles. On en espère que de proche en proche, les principaux intéressés que sont les deux princes en viendront eux aussi à se sacrifier, en faisant mourir non eux-mêmes mais leurs griefs sur l'autel du salut commun : vaincre son cœur (B. 1409). Leur refus de changer n'en est que plus insupportable.

Nous avons vu que l'exorde ou la narratio sont des préparations de l'argumentation. De même, Jocaste est donc orateur en ce qu'elle prépare de longue main, très en amont, ce qui rendra efficace son discours et cérémonie de conciliatio. En orateur prudens, elle sait aussi saisir le bon moment ou kairos, celui où les esprits seront enfin prêts à recevoir ses paroles. Sa façon de brusquer les choses n'est pas une maladresse, cela rappelle la métaphorisation militaire, très habituelle en rhétorique. De même que la stratégie inclut la tactique, le rythme de la progression passe par des problèmes de tempo et d'intuition du moment favorable, donc par des moments d'accélération. Comme Burrhus, qui est encore plus nettement militaire qu'elle, Jocaste est le personnage qui aura fait ce qu'on pouvait humainement faire, par les moyens humains de la parole, non sans invoquer comme il se doit l'aide divine. Loin d'être des optimistes, tous deux sont des pessimistes actifs.

Dans Britannicus, si on n'a pas un tel travail en amont que celui de Jocaste, l'habitus est là encore un élément-clé. Néron a semblé jusque là le contraire d'un cœur de fer. D'un point de vue rhétorique, il est même très bonne pâte, très influençable, puisque chaque discours le fait changer de disposition. Mais le spectateur sait que ce sont des dispositions passagères. Sa mère Agrippine a l'habitude de son habitus de gentil garçon : il suffisait qu'elle parle pour qu'il change d'attitude, «J'ai parlé, tout a changé de face» (1583). Junie dans sa scène de pitié fait un pari sur son habitus de prince apaisable. Burrhus enfin espère pouvoir se fonder sur les « trois ans de vertus » (462) de Néron sous son préceptorat, que Néron lui-même vit comme un fardeau. L'une des abominations de la pièce est que toute la théorie pédagogique de l'habitus s'écroule. La pratique répétée de la vertu, dont la vertu de clémence (1367-1372), doit dans la théorie faire passer d'une disposition passagère à une disposition stable ou habitus. Mais les institutions du Prince savent bien que l'adolescence est le moment par excellence du changement d'habitus, celui où il faut choisir la voie du vice ou celle de la vertu, selon le Y pythagoricien ("C'est à vous de choisir »,1339). Une fois qu'on sera engagé dans la branche vertueuse du Y, ensuite la voie sera aisée, on sera dans la firma facilitas de l'habitus. Néron au contraire s'engage dans la voie du vice, aisée au début, et enfer ensuite. Du point de vue de l'habitus, Burrhus a de longue date compris son élève, il est un éducateur prudens. Agrippine n'a rien compris au changement souterrain en cours. Junie, elle, en a l'intuition, que lui inspire Amour. Ces trois personnages incarnent aussi trois figures d'orateur, et autant d'échecs. Junie est la rhétorique naturelle de la sincérité. À l'extrême inverse, Agrippine est l'orateur qui croit l'ars toute puissante, la prend pour une magie (noire) qui va forcer la liberté de l'auditeur, avec les grands moyens d'un pathos exacerbé. Burrhus est le juste milieu entre nature et artifice. Tout 
à la fois Romain et militaire, il recourt aux arguments et aux larmes, au logos comme au pathos. Plus chanceux que Jocaste, ses larmes touchent Néron (1381). Provisoirement: car pas plus que Jocaste il n'est aidé du Ciel. Dans un monde dont la grâce est absente, le seul qui puisse réussir est un flatteur comme Narcisse, image du sophiste qui est en soi antinomique de toute idée d'orateur. La bonne rhétorique est le contraire de l'art de flatter.

40 Terminons sur la conciliatio par la description chez Vossius de sa deuxième sorte (Rhetorice contracta, sa p. 192). Quoique très brève, elle va nous permettre de confronter le discours de conciliatio avorté de La Thébaïde et celui de Burrhus, canonique et apparemment efficace ${ }^{16}$ :

§5. Pour réconcilier des partis qui s'affrontent, voici comment procéder. Nous montrons que toute offense doit être effacée : d'une part parce que c'est plus utile et plus sûr, et d'autre part, parce que c'est aussi plus honorable [honestius]. Les passions qui règnent ici sont l'espoir et la crainte.

§ 6. Mais autant il est facile d'exciter les gens à la sédition [concitatio], autant il est difficile de les réconcilier. Car la plupart des gens sont trop attachés à leur colère et au souvenir de l'offense reçue.

"L'utile et l'honnête » (titre du chapitre III, 1 des Essais) sont ici le couple canonique du délibératif, ce grand genre dont relève la conciliatio.

41 L'«utile» et le sûr désignent les intérêts bien compris. Cela renvoie au réalisme politique d'un Pharnace, qui lui aussi agite le pathos de la crainte, sinon son pendant l'espoir, celui de voir nos affaires prospérer ${ }^{17}$. Chez Tite-Live, c'est le réalisme du chef albain qui propose au roi romain le combat des Horace et des Curiace : craignons notre ennemi commun, les Étrusques, qui ne feront qu'une bouchée de nous quand nos deux armées se seront entre-déchirées. Le discours de Burrhus commence de même par là : il est contre vos intérêts de faire mourir Britannicus, ses amis ou clan voudront le venger, donc pathos de la crainte (1342-1354). Narcisse comme en réponse fera de même (1402-1408 et 1661-1670) : Britannicus vivant voudra se venger de son emprisonnement et de votre projet de l'assassiner. Burrhus et Narcisse raisonnent tous deux sur le devoir de vengeance, et en somme l'argument du second contrebalance celui $d u$ premier.

L'« honnête " de son côté désigne l'honorable, ou de façon plus claire la gloire ou le beau moral, gloria ou pulchrum, termes qui en pratique varient souvent celui d'honestum. Chez Vossius et Burrhus, la combinaison harmonieuse des deux est de nouveau une forme de hiérarchisation. Loin qu'il y ait antinomie entre, en termes modernes, les intérêts et la morale, le monde rhétorique d'Aristote à Cicéron y voit une complémentarité. De façon idéale, la gloire n'exclut pas les intérêts bien compris, et réciproquement. Un monde qui sépare les deux est un monde où précisément ne règne pas la parole, avec sa dimension religieuse de lien. La rhétorique joint l'utile au beau. Il est beau, et utile, de se réconcilier. Si de façon cynique on disjoint les deux, le cynisme retentit sur les deux branches ainsi disjointes. La quête de l'intérêt personnel ou partisan paraîtra illégitime, et la quête de la gloire une forme insupportable de vanité, des formules creuses pour masquer la poursuite de ses intérêts (de la « rhétorique » en son sens négatif actuel). Or ce cynisme est le pain quotidien du monde "réaliste », qui a beaucoup de mal à imaginer la visée idéale d'une continuité entre intérêt et morale, tout comme d'ailleurs entre essence et apparence. Dans l'idéal, le beau est l'ornement du bien, sa visibilité, et non le masque trompeur du mal. À l'inverse, aux « réalistes » ou plutôt cyniques seule la quête de l'utile paraît « réaliste », tout comme la laideur. 

Néron de Britannicus sont dans un monde disjoint, un monde profondément non rhétorique. La Thébaïde est une guerre fratricide, comme Albe et Rome des origines, mais sans ennemi extérieur aux deux partis. Jocaste ne peut agir sur les deux frères avec le levier si puissant et pathétique de la crainte d'un péril commun. Nous sommes dans une sorte de vacuum politique, où le levier de l'«utile " est indisponible, et où celui de la gloire est inaudible. Cela annonce la manière dont le sérail de Bajazet est dans le vide, dans une bulle, seul le grand vizir Acomat n'y perd pas de vue le monde extérieur, et lui qui a tellement les pieds sur terre a l'air dans la pièce d'être un extraterrestre, comme d'ailleurs Pharnace. Dans Britannicus, Burrhus ajoute au levier de l'utile celui de l'honestas (1337-1385). Il a bien compris que Néron y est plus sensible, il le lit dans ses yeux ("Mais je vois »,1381). Et Néron auparavant a déjà donné comme raisons « Ma gloire » avant « ma sûreté » (1324; et 1427-1431). Burrhus dans sa réponse s'appuie sur la vision classique qu'a Cicéron de la gloire, selon laquelle l'éloge est le salaire de la vertu: "la gloire est la louange qui s'attache aux belles actions et aux grands services rendus à la patrie, sanctionnés à la fois par le témoignage de l'élite et par celui de la multitude » (Phil. I, 29). Néron n'est pas loin de se laisser gagner par cette noble vision de la maîtrise ("vous êtes encor maitre ", 1339), mais sous influence de Narcisse il va ensuite confondre comme Marc Antoine la gloire et la maitrise avec le pouvoir (Cicéron, Phil. I, 33) :

[...], ignorant le véritable chemin de la gloire, tu crois qu'il est glorieux pour toi d'avoir à toi seul plus de pouvoir que tous les autres et que tu aimes mieux être craint de tes concitoyens qu'être aimé d'eux. Si telle est ta pensée, tu ignores complètement le chemin de la gloire : être cher à ses concitoyens, bien servir la patrie, être loué, honoré, aimé, voilà qui est glorieux.

Nous retrouvons la problématique de l'amour du peuple pour son prince, cet ornement ou luxe en fait si nécessaire. Du reste, Néron parle comme Polynice, non Étéocle (« Suisje leur empereur seulement pour leur plaire?»,1336).

Le programme rhétorique de Britannicus est ainsi donné par un vers comme "Quoi ! toujours enchaîné de ma gloire passée » (1332), qui annonce la reformulation finale du concept de gloire. Néron libéré de ses chaînes et de son «joug» $(468,1678)$ va supporter de voir les Romains " au joug », « enchaînés » (1443-1444). Il voit la louange des autres comme un asservissement, comme sa servitude volontaire à lui, et il s'en libère triomphalement, à ses yeux, se sentant par là enfin magnanime, en pleine grandeur d'âme, s'imaginant enfin n'être plus influencé, lui l'influençable. Mais dans cette conquête de "liberté » et de " maîtrise ", les spectateurs voient, avec terreur et pitié, l'asservissement à sa passion du pouvoir et à son orgueil. Pour parler comme le roi Philippe à ses deux fils, « rien ne lui est cher ni sacré : un désir insatiable de régner a étouffé dans son cœur tout autre sentiment ${ }^{18}$.

46 Au terme du parcours, nous pouvons revenir sur les notions initiales d'« échec " et surtout de «bonne" rhétorique. De la rhétorique nous aimerions bien aujourd'hui pouvoir donner une description purement technique et par là amorale, qui égaliserait Burrhus et Narcisse. Racine comme son époque n'imagine pas disjoindre morale et technique, le vir bonus et le dicendi peritus de Quintilien. Son absence de gêne vient, en un mot, de ces évidences qu'étaient alors pour tout le monde la religion et la dimension de lien qu'a la parole, d'où la résonance que prend ce mot de mystère. 
Quoi qu'il en soit, de La Thébaïde à Britannicus, on a une gradation sensible. La ressemblance des deux pièces est que la barre de la réussite et donc de l'échec y est placée très haut. Le but n'est pas d'obtenir un changement ponctuel du Prince (ou des Princes), mais un changement durable, autrement dit une conversion totale. Les discours de Junie ou de Burrhus obtenant la grâce de Britannicus sont des réussites passagères, ce qui sert de faire-valoir à leur échec de fond. Les deux pièces tirent parti de leur longueur même pour réintroduire le temps long dans la discussion sur l'échec ou la réussite. D'où l'importance de la notion d'habitus. La réussite serait ou le changement d'habitus des "cœurs de fer ", ou le maintien de Néron dans son habitus vertueux. D'une certaine manière, on retrouve ici la négation de toute idée d'habitus dans le bien, par un Luther puis la Réforme, pour qui la seule stabilité est celle dans le mal, tout le reste venant de la grâce divine. D'où aussi, dans cette temporalité longue des pièces, l'importance des petits pas qu'obtient Jocaste. Une concession mineure comme celle du délai accordé est déjà quelque chose, cette bonne disposition passagère est peut-être le signe annonciateur d'un changement plus grand, plus durable aussi. Jocaste échoue, mais sa grandeur est à la mesure de la grandeur de son espoir, c'est un personnage profondément religieux.

La différence essentielle entre les deux pièces est que $L a$ Thébaïde a de la rhétorique une vision non problématique. La parole y est du côté de la paix (et des femmes), et les Princes, de la guerre. C'est du reste leur grandeur à eux. Dès le début ils ont décidé que seules la guerre ou la mort de l'un des deux pouvaient les sortir de la crise. En guerriers, ils n'ont que faire de toutes ces paroles de paix et de femmes. L'échec rhétorique ne nous apprend alors sur la rhétorique elle-même que ce que nous savions déjà : que les tenants de la force ne croient pas à la force de la parole, ou encore que le monde païen n'est pas le monde chrétien.

Britannicus est bien plus dérangeant, tant tout y est perverti. Le mystère atroce du banquet néronien est la moquerie non moins atroce du mystère de toute parole, de même que l'éducation de Néron est le cauchemar de tout pédagogue. Mais cette moquerie met au défi de dire ce qui fait la bonne rhétorique. Le mystère de celle-ci reste un mystère. Le paradoxe éternel de la parole qui veut changer autrui est de prétendre à la fois respecter pleinement sa liberté et la contraindre de fait par l'appel à ce qui dépasse l'auditeur, que ce soit « le Ciel » ou Rome, Amour ou Nature, une religion divine ou civile. C'est une contrainte libre, une douce violence, un soft power. L'art de Racine ne consiste pas à lever un tel mystère, il nous donne à l'entrevoir et percevoir, ce qui est déjà considérable.

\section{NOTES}

1. Pour un exemple de discours où l'orateur mime ainsi un dialogue fait d'objections et de réponses au style direct (une sermocinatio), voir Cicéron, $1^{\text {re }}$ Philippique, § 20. 
2. On remarque un phénomène très habituel: le groupement de quatre vers. Quatre pour l'exorde, l'argument $\mathrm{n}^{\circ} 2$, la conclusion; huit vers pour l'argument $\mathrm{n}^{\circ} 1$ (en somme, deux fois quatre). Cela seul pousse à voir deux objections à 355-358.

3. Monime à Xipharès, M. 133-162 ; Junie à Britannicus, B. 637-658. Même lieu solitudo ou absence d'appuis : B. 655, «Britannicus est seul », par parallèle avec Néron si entouré ; M. 133-138, comme Iphigénie 937-940 et Andromaque 928-931. Je me permets de renvoyer à mon article « Les "lieux" de la pitié dans Athalie ", Styles, genres, auteurs, $\mathrm{n}^{\circ} 3,2003$, p. 91-103.

4. Trad. Ch.-É. Ruelle, Paris, Le Livre de Poche, 1991, p. 189. Cf. la traduction et paraphrase de Bauduyn de La Neufville, La rhétorique royale d'Aristote, Paris, André Boutonné, 1669, ad loc. (je souligne) : « Mais ce que nous trouvons de plus insupportable, et qui nous emporte davantage, c'est quand nous recevons une injure, et quand nous sommes offensés par nos amis, contre lesquels nous nous fâchons davantage, que contre d'autres personnes, parce que l'offense est d'autant plus grande, que l'offenseur est cher; et il est contre toute sorte de justice de recevoir du déplaisir, et un mauvais office de la part de ceux dont nous ne devons attendre que des bienfaits, et des marques d'une mutuelle bienveillance. » Tout ceci est évidemment, dans la bouche de Créon, à double entente, puisqu'il est amoureux d'Antigone, avec son fils comme rival. La scène de colère se déploie de façon officielle, avec des doubles sens galants.

5. Essais, II, 12, éd. Villey-Saulnier, Paris, PUF, 1965, p. 546 ; éd. Naya, Paris, Gallimard (Folio), 2009, II, p. 314. Montaigne a écrit « un philosophe imprémédité ».

6. Jocaste à Étéocle, 146, «Au moins consolez-moi de quelque heure de paix. / Accordez cette grâce aux larmes d'une mère »; et Antigone à Polynice, 539, « Seulement quelques jours souffrez que l'on vous voie / Et donnez-nous le temps de chercher quelque voie / [...] Pouvez-vous refuser cette grâce légère / Aux larmes d'une sœur, aux soupirs d'une mère ? » Imitation de Didon face à Énée décidé à la quitter, Énéide, IV, 429-430, extremum hoc miserae det munus amanti : exspectet. Ferrazzi en 1694 paraphrase en remque exiguam peto, et l'abbé Desfontaines traduit en 1743 (Paris, Quillau père) "Qu'il accorde au moins à son amante une grâce légère : c'est d'attendre pour s'embarquer que la saison soit plus favorable».

7. «Vos libertés", 278 ; "Ses superbes discours», 292 ; et 992 , «le plus furieux passe pour magnanime ». Dans B., Burrhus a « la liberté d'un soldat » (173-174), et de la « hardiesse » (1643). La compétition pour la « vraie " grandeur d'âme fait rage, chez Racine comme dans les Essais.

8. «Qui ne sait point [...] que si de cette couronne, / Que sa tige illustre lui donne, / Les lois ne l'eussent revêtu, / Nos peuples d'un juste suffrage / Ne pouvaient sans faire naufrage / Ne l'offrir point à sa vertu? » (Ode sur l'attentat commis en la personne de sa majesté..., v. 31-40). Idem pour le Louis XIV du début du règne, ou le jeune Louis XV, surnommé le «bien aimé ».

9. Vossius, Rhetorice contracta, II, chap. XXII, $\$ 2$ (dans l'éd. 1660 donnée ci-après dans l'Atelier, p. 191).

10. XL, 46, 1-15; chez Ferrazzi, discours 165. Je cite la traduction Nisard (Paris, 1864). Sur les 180 discours de Tite-Live que Ferrazzi analyse, il en nomme seulement trois des conciliationes : le tout premier (I, 23), le 21 ${ }^{\mathrm{e}}$ (III, 67-68) et celui-ci.

11. Pour reprendre le sous-titre d'I. Rosier-Catach, La parole efficace. Signe, rituel, sacré, Paris, Seuil, 2004.

12. $§ 15$; voir aussi ses $\S 33-35$ sur le « Qu'ils me haïssent pourvu qu'ils me craignent ». La $1^{\text {re }}$ Philippique était un des grands classiques de la dénonciation de la tyrannie, du mauvais roi.

13. Vossius, Institutionum libri sex, II, 3 (dans l'éd. donnée ci-après dans l'Atelier, p. 216).

14. Vulgatum illud, quia uerum erat, in prouerbium uenit, amicitias immortales, <mortales> inimicitias debere esse. C'est alors que la foule le relaie par «mille prières ». On peut penser que inimicitia est ici un euphémisme pour odium: une manière de l'atténuer et de rendre ainsi possible sa disparition.

15. Et Vossius (Rhetorice contracta, II, chap. XXII, § 5) : «Ostendimus, offensam omnem obliterari debere », notre discours de réconciliation dira « On doit oublier toute offense reçue ». La colère 
par exemple étant un devoir de vengeance, de punition, le conflit entre deux pathè (colère ou apaisement, punition ou clémence) est un conflit entre deux devoirs, deux légitimités ; pas entre la « passion » et la « raison ». D'où les binômes d'Aristote.

16. Discours confirmé comme conciliatio par le « on nous réconcilie » du vers 1400 . Il s'en déduit que le discours de Narcisse, vu de haut (IV, 4, 1401-1479), est l'inverse : un discours de concitatio, qui excite Néron à secouer le joug. Il s'en déduit aussi que le discours global de Polynice à Jocaste est un discours de concitatio : s'affirmer soi, c'est affirmer comme inoubliable l'offense reçue.

17. Dans les trois discours de conciliation chez Tite-Live selon Ferrazzi, l'espoir est présent pour le deuxième, dans sa péroraison («spes certae victoriae ", dit Ferrazzi) : avec notre discorde entre patriciens et plébéiens, les ennemis de Rome sont à nos portes ; mais réconcilions-nous, et la victoire ne peut nous échapper.

18. "Nihil cari, nihil sancti est. In omnium vicem regni unius insatiabilis amor successit": TiteLive, XL, 18 (chez Ferrazzi, discours 161). C'est la chute de tout un passage (depuis le $\S 11$ ), lequel est un topo classique sur l'avantage de la réconciliation entre frères ennemis. À noter que TiteLive voit ici dans le frère pro-romain (l'équivalent de Pharnace) le héros positif, et dans son frère anti-romain le héros négatif. - Pour finir sur le discours de conciliatio de Burrhus, celui-ci ne dit pas à la Vossius qu'il faut «oublier toute offense reçue ", parce qu'à ses yeux il n'y a pas eu d'offense, et que Britannicus est innocent. Mais cette innocence même (1386), prudemment il ne la dit que quand il pense avoir cause gagnée.

\section{AUTEUR}

\section{FRANCIS GOYET}

Univ. Grenoble-Alpes, RARE - Rhétorique de l'Antiquité à la Révolution 